\title{
Effects of ageing and smoking on SP-A and SP-D levels in bronchoalveolar lavage fluid
}

\author{
T. Betsuyaku*, Y. Kuroki", K. Nagai*, Y. Nasuhara*, M. Nishimura*
}

Effects of ageing and smoking on SP-A and SP-D levels in bronchoalveolar lavage fluid. T. Betsuyaku, Y. Kuroki, K. Nagai, Y. Nasuhara, M. Nishimura. (C)ERS Journals Ltd 2004.

ABSTRACT: Surfactant protein (SP)-A and SP-D are collagen-like glycoproteins that are synthesised in the distal pulmonary epithelium. This study examined the effects of ageing and long-term smoking on SP-A and SP-D in the lungs. The possible links to the development of pulmonary emphysema were also investigated.

Sequential lavage was performed in young and middle-aged or elderly nonsmokers and asymptomatic current smokers with various smoking histories. Middle-aged or elderly smokers were further categorised according to the presence of emphysema by high-resolution computed tomography. Levels of SP-A and SP-D in bronchial lavage (BL) fluid and in bronchoalveolar lavage (BAL) fluid were quantified by ELISA.

Significant decreases in SP-A were seen with age in nonsmokers in BL fluid, but not in BAL fluid. Middle-aged or elderly smokers with emphysema had lower levels of SP$A$ in both BL and BAL fluids when compared with young subjects, and in BL fluid when compared with middle-aged or elderly smokers without emphysema. SP-D did not change with age alone, however, it was decreased in middle-aged or elderly smokers when compared with similarly aged nonsmokers.

In conclusion, surfactant protein-A may decrease with age alone or due to the cumulative effects of long-term smoking and development of emphysema, while surfactant protein-D decreases due to long-term smoking.

Eur Respir J 2004; 24: 964-970.
*First Dept of Medicine, Hokkaido University School of Medicine and \# Dept of Biochemistry, Sapporo Medical College, Sapporo, Japan.

Correspondence: T. Betsuyaku, First Dept of Medicine, Hokkaido University School of Medicine, Kita-15, Nishi-7, Kita-ku, Sapporo 060-8638, Japan.

Fax: 81117067899

E-mail: bytomoko@med.hokudai.ac.jp

Keywords: Ageing, chronic obstructive pulmonary disease, collectin, pulmonary emphysema, sequential bronchoalveolar lavage, smoking

Received: June 12004

Accepted after revision: August 62004

This research was supported by the Respiratory Failure Research Group of the Ministry of Health, Labor, and Welfare of Japan (to M. Nishimura) and scientific research grant 14570532 from the Ministry of Education, Science, Culture and Sports of Japan (to $\mathrm{T}$. Betsuyaku).
The elderly are particularly vulnerable to smokingassociated respiratory diseases. The ratio of smokers is still high among the worldwide middle-aged or elderly population and they usually have long smoking histories. However, the cumulative effects of ageing and long-term smoking on pulmonary defence mechanisms remain to be fully elucidated. The epithelium is the first line of defence against inhaled noxious agents, such as cigarette smoke, and microorganisms in the lungs. Surfactant protein (SP)-A and SP-D are collagen-like glycoproteins belonging to the collectin class of C-type lectins, and they are synthesised in alveolar type II cells and nonciliated bronchiolar cells of the distal pulmonary epithelium [1]. Assessment of the physiological importance of the pulmonary collectins in innate immunity has recently become feasible through the development of genetically engineered experimental animal models [2]. While binding of collectins to microorganisms may facilitate microbial clearance through aggregation or other direct effects on the micoroorganisms, SP-A and SP-D also have the capacity to modulate leukocyte function and to enhance the killing of microorganisms [3]. Surprisingly, mice deficient in SP-D, but not SP-A, develop progressive emphysema with age [4], thus highlighting the connection between the lack of an epithelial cell-derived protein and elastin degradation through the unwanted activation of alveolar macrophages in the lung, in which ageing might be an accelerating factor.

It has been established that alveolar type II cells and nonciliated bronchial cells are the major cells producing SP-A and SP-D in the lungs [5, 6]. The distribution of SP-A and SP$\mathrm{D}$ might be regulated differently in particular microenvironments along the airways and thus may serve as markers of local epithelial cell function. The current study therefore investigated whether age- and/or smoking-associated changes might occur independently with regard to SP-A and SP-D in airways and in alveolar spaces and whether these changes would help to explain the reduced pulmonary host defences and/or increased susceptibility to destructive changes in the extracellular matrix of aged smokers. In this study, sequential lavage was performed in young and middle-aged or elderly non- and current smokers, and the levels of SP-A and SP-D were assessed in bronchial lavage (BL) fluid, which contains components from the airway, and in bronchoalveolar lavage (BAL) fluid, which is representative of components in alveolar space. Data was then analysed on the middle-aged or elderly current smokers exhibiting emphysematous changes on highresolution computed tomography (HRCT) scans in order to assess the possible link between these proteins and the development of early emphysema.

\section{Materials and methods}

\section{Subjects}

Life-long nonsmokers consisted of 15 young males and seven middle-aged or elderly males. Current smokers were 20 
young males who had smoked for $<3$ yrs and 62 middle-aged or elderly subjects, including six females, with smoking histories of $>20$ yrs. Former smokers were excluded from this study. For middle-aged or elderly smokers, HRCT scans were performed in order to screen for emphysematous changes and they were then classified to the presence or absence of emphysematous changes as previously described [7]. Briefly, three pulmonary physicians, who were blind to any information about individual patients, independently evaluated the presence of emphysematous changes in all serial horizontal slices on HRCT scans. We defined subjects as having emphysema $(+)$, regardless of the magnitude or severity of disease, on the basis of agreement all physicians. All the other subjects were defined as emphysema (-). Pulmonary function tests were performed for vital capacity and forced expiratory volume in one second (FEV1) for all the subjects. Spirometory and expiratory flow rates were measured on a rolling seal spirometer (CHESTAC-55V; CHEST Co., Tokyo, Japan) according to the standard techniques recommended by the American Thoracic Society [8]. The best values of three acceptable curves were used for the analysis. None of the subjects had respiratory symptoms or comorbidties, and had been free of acute respiratory infections for the preceding 2 months. None of the subjects reported occupational exposure or history of lung disease. Written informed consent was obtained from each subject, and the Ethics Committee of Hokkaido University School of Medicine approved the study protocols. Most of the middle-aged or elderly current smokers had acted as subjects in previous studies from the First Dept of Medicine, Hokkaido University School of Medicine [9, 10].

\section{Sequential bronchoalveolar lavage}

Patients and healthy volunteers underwent sequential BAL, as detailed elsewhere, in an attempt to separately collect materials from the large and peripheral airways [10]. Briefly, the right middle lobe or the left counterpart of each subject was lavaged with $50-\mathrm{mL}$ aliquots (total volume: $200 \mathrm{~mL}$ ) of sterile $0.9 \%$ saline at room temperature through a wedged flexible fiberoptic bronchoscope (Olympus BF-B3R, Tokyo, Japan). The fluid recovered after the first $50-\mathrm{mL}$ aliquot was used as BL fluid containing cells and material from the large airways. The remaining lavage fluid was pooled and used as BAL fluid. Recovered BL fluid and BAL fluid were separately filtered through several layers of gauze in order to remove excess mucus and debris, and was then centrifuged at $1,500 \mathrm{rpm}$ for $5 \mathrm{~min}$ at $4^{\circ} \mathrm{C}$ in order to separate the supernatant from the cells. Aliquots of the supernatant $(1 \mathrm{~mL})$ were immediately frozen and stored at $-70^{\circ} \mathrm{C}$ prior to assay. Cell pellets were counted in a haematocytometer, and Wright-Giemsa stain smears served to identify differential profiles after cytospin preparation. Differential counts were performed by examining $\geqslant 200$ cells using a standard light microscope as previously described [7]. Albumin in unconcentrated BAL fluid supernatant was measured by laser nephelometry for all the subjects as described previously [7].

\section{ELISA for surfactant protein-A in bronchial lavage and bronchoalveolar lavage fluids}

SP-A levels in unconcentrated lavage fluids were determined by ELISA using solid-phase monoclonal antibody PC6 and HRP-conjugated monoclonal antibody PE10 as previously described [11]. The assay was able to detect between $2.0-200 \mathrm{ng} \cdot \mathrm{mL}^{-1}$ of SP-A. Equivalent aliquots of lavage fluid were used for the ELISA. The assay was performed in duplicate and data were expressed as the mean of duplicate samples. Samples were mixed vigorously prior to measurement in order to avoid precipitation of aggregated SP-A in lavage fluids [12].

\section{ELISA for surfactant protein-D in bronchial lavage and bronchoalveolar lavage fluid}

SP-D levels in unconcentrated lavage fluids were measured by sandwich ELISA using monoclonal antibody 7C6 and HRPconjugated monoclonal antibody $6 \mathrm{~B} 2$ as previously described [13]. The sensitivity of this assay was $3.1 \mathrm{ng} \cdot \mathrm{mL}^{-1}$. Equivalent aliquots of lavage fluid were used for the ELISA. The assay was performed in duplicate and data were expressed as the mean of duplicate samples.

\section{Western blotting}

Unconcentrated BAL fluid $(10 \mu \mathrm{L})$ was denatured and reduced in the presence of 2-mercaptoethanol, and was then electrophoresed in 10\% sodium-dodecylsulphatepolyacrylamide gels using pre-stained molecular weight markers (Bio-Rad, Hercules, CA, USA). Proteins were then transferred to PVDF membranes (Millipore, Bedford, MA, USA), using a semi-dry transfer cell (AE-6677, ATTO, Tokyo, Japan). Nonspecific binding sites were blocked with Tris-buffered saline-containing $0.1 \%$ Tween 20 (TBS-T) and $5 \%$ nonfat dried milk for $1 \mathrm{~h}$ at room temperature. Membranes were incubated with a monoclonal antibody against SP-A (PC6) at $5 \mu \mathrm{g} \cdot \mathrm{mL}^{-1}$ or with a monoclonal antibody against SP-D (6B2) at $10 \mu \mathrm{g} \cdot \mathrm{mL}^{-1}$ overnight at $4^{\circ} \mathrm{C}$. After three washes with TBS-T, the membranes were incubated in horseradish peroxidase-conjugated anti-mouse immunoglobulin or anti-rabbit immunoglobulin at 1:10,000 dilution (DAKO). Sites of antibody binding were visualised using the ECL Plus $\mathbb{R}$ Western blotting detection system (Amersham, Aylesbury, UK).

\section{Statistical analysis}

All values were expressed as mean \pm SEM unless otherwise stated. Statistical analyses were performed using one-factor ANOVA among the five groups followed by Fisher's test. For comparison between BL and BAL fluid, data were analysed by paired t-test. The Spearman's rank correlation was calculated to assess the correlation between data. In all tests, p-values $<0.05$ were considered statistically significant.

\section{Results}

\section{Characteristics of the subjects}

Clinical characteristics and pulmonary function data are summarised in table 1. Age of the young subjects ranged from 18-29 yrs, and that of the middle-aged or elderly subjects ranged from 41-77 yrs. There were no significant differences in age or pack-yrs of smoking between middle-aged or elderly smokers with or without emphysematous changes on HRCT scans. Pulmonary function data revealed that FEV1/forced vital capacity was slightly, but significantly lower in the group with emphysema than in the group without emphysema $(p<0.05)$. BAL findings are shown in table 2. The total recovery rate for middle-aged or elderly smokers with emphysema was significantly lower compared to the others 
Table 1.-Characteristics of subjects

\begin{tabular}{|c|c|c|c|c|c|}
\hline & \multicolumn{2}{|c|}{ Nonsmokers } & \multicolumn{3}{|c|}{ Current smokers } \\
\hline & \multirow[t]{2}{*}{ Young } & \multirow[t]{2}{*}{ Middle-aged or elderly } & \multirow[t]{2}{*}{ Young } & \multicolumn{2}{|c|}{ Middle-aged or elderly } \\
\hline & & & & Emphysema (-) & Emphysema (+) \\
\hline Subjects n (female) & $15(0)$ & $7(0)$ & $20(0)$ & $26(1)$ & $36(5)$ \\
\hline Age yrs & $22 \pm 1$ & $63 \pm 3$ & $23 \pm 1$ & $54 \pm 2$ & $56 \pm 2$ \\
\hline Pack-yrs & 0 & 0 & $5 \pm 1$ & $49 \pm 4$ & $45 \pm 4$ \\
\hline VC $\%$ pred & $97 \pm 3$ & $122 \pm 8$ & $96 \pm 3$ & $109 \pm 3$ & $109 \pm 3$ \\
\hline FEV1 $\%$ pred & $82 \pm 3$ & $124 \pm 8$ & $86 \pm 3$ & $103 \pm 3$ & $93 \pm 3$ \\
\hline FEV1/FVC \% & $93 \pm 2$ & $84 \pm 2$ & $90 \pm 2$ & $79 \pm 1$ & $71 \pm 2$ \\
\hline
\end{tabular}

Data are presented as mean \pm SE. VC: vital capacity; FEV1: forced expiratory volume in one second; FVC: forced vital capacity.

( $\mathrm{p}<0.05$, respectively), and it was also lower for middle-aged or elderly smokers without emphysema compared with young and middle-aged or elderly nonsmokers $(\mathrm{p}<0.05$, respectively). There were no differences in the concentration of albumin in BAL fluid among the five groups. The percentage of macrophages and neutrophils in BAL fluid did not differ among the three groups of current smokers.

\section{Surfactant protein-A in bronchial lavage and bronchoalveolar lavage fluids}

The concentration of SP-A in lavage fluids was compared among the five groups (fig. 1). A significant decrease in SP-A was seen with age in BL fluid from nonsmokers, but no decrease was seen in BAL fluid. A significant decrease in SP-A both in BL fluid and in BAL fluid was observed in middleaged smokers with emphysema when compared with that from young nonsmokers and BL fluid from young smokers. In addition, the levels of SP-A in BL fluid differed significantly between the two groups of middle-aged or elderly smokers with and without emphysema.

\section{Surfactant protein-D in bronchial lavage and bronchoalveolar lavage fluids}

In contrast to SP-A, smoking habits diversely affected levels of SP-D in lavage fluids with age (fig. 2). Both groups of middle-aged or elderly current smokers, irrespective of the presence of emphysema, had significantly lower levels of SP-D in BL fluid when compared with young and middle-aged or elderly nonsmokers, as well as young current smokers. Similar differences were also seen in BAL fluid. However, no significant differences were observed in the levels of SP-D in $\mathrm{BL}$ or BAL fluid between the two young groups. There were no significant differences in the levels of SP-D in BL or BAL fluid between the middle-aged or elderly smokers with and without emphysema.

Comparisons of bronchial lavage and bronchoalveolar lavage fluid in the levels of surfactant protein-A or surfactant protein-D

Levels of SP-A and SP-D in BL fluid were generally higher when compared with those in BAL fluid (SP-A: $4,043 \mathrm{ng} \cdot \mathrm{mL}^{-1}$ versus 2,785 , and SP-D: $996 \mathrm{ng} \cdot \mathrm{mL}^{-1}$ versus 593, $\mathrm{p}<0.05$, respectively). SP-D levels in $\mathrm{BL}$ fluid were strongly correlated with those in BAL fluid $(\mathrm{p}<0.01, \mathrm{r}=0.67)$ (fig. 3b), while a weaker but significant correlation was seen for SP-A in the two fluids $(\mathrm{p}<0.01, \mathrm{r}=0.07)$ (fig. $3 \mathrm{a})$.

\section{Molecular status of surfactant protein- $A$ and surfactant protein-D in bronchoalveolar lavage fluid}

The molecular states of SP-A and SP-D in BAL fluid were compared under reducing conditions between young nonsmokers and middle-aged or elderly current smokers with emphysema. In BAL fluids from young nonsmokers (fig. 4a, lanes 1-4) a heavy band at $\sim 36 \mathrm{kDa}$ and an additional $\sim 62 \mathrm{kDa}$ band were detected using the monoclonal antibody PC6. In BAL fluids from middle-aged or elderly current smokers with emphysema (fig. 4a, lanes 5-8) the $\sim 36 \mathrm{kDa}$ band was also present, but was much less prominent than in BAL fluid from young nonsmokers, and the $\sim 62 \mathrm{kDa}$ band was barely visible. The monoclonal antibody $6 \mathrm{~B} 2$ detected SP-D bands at $\sim 43 \mathrm{kDa}$ (monomeric subunits), and at $\sim 50 \mathrm{kDa}$ in all but one subject (lane 1) under reducing conditions (fig. 4b). BAL fluids from middle-aged or elderly current smokers with emphysema have decreased SP-D in

Table 2. - Characteristics of bronchoalveolar lavage fluid

\begin{tabular}{|c|c|c|c|c|c|}
\hline & \multicolumn{2}{|c|}{ Nonsmokers } & \multicolumn{3}{|c|}{ Current smokers } \\
\hline & \multirow[t]{2}{*}{ Young } & \multirow[t]{2}{*}{ Middle-aged or elderly } & \multirow[t]{2}{*}{ Young } & \multicolumn{2}{|c|}{ Middle-aged or elderly } \\
\hline & & & & Emphysema (-) & Emphysema $(+)$ \\
\hline Total recovery rate $\%$ & $75 \pm 4$ & $71 \pm 4$ & $63 \pm 3$ & $48 \pm 3$ & $38 \pm 3$ \\
\hline Total cells $\times 10^{4} \cdot \mathrm{mL}^{-1}$ & $8 \pm 1$ & $13 \pm 1$ & $19 \pm 2$ & $32 \pm 6$ & $29 \pm 5$ \\
\hline Viable cells $\%$ & $96 \pm 1$ & $97 \pm 1$ & $95 \pm 1$ & $93 \pm 1$ & $86 \pm 2$ \\
\hline Macrophages \% & $84 \pm 2$ & $81 \pm 5$ & $95 \pm 1$ & $93 \pm 1$ & $92 \pm 1$ \\
\hline Neutrophils \% & $1.1 \pm 0.3$ & $0.3 \pm 0.1$ & $0.8 \pm 1.2$ & $1.8 \pm 0.7$ & $1.4 \pm 0.3$ \\
\hline Albumin $\mu \mathrm{g} \cdot \mathrm{mL}^{-1}$ & $47 \pm 7$ & $55 \pm 10$ & $32 \pm 2$ & $35 \pm 3$ & $52 \pm 8$ \\
\hline
\end{tabular}

Data are presented as mean \pm SE. 

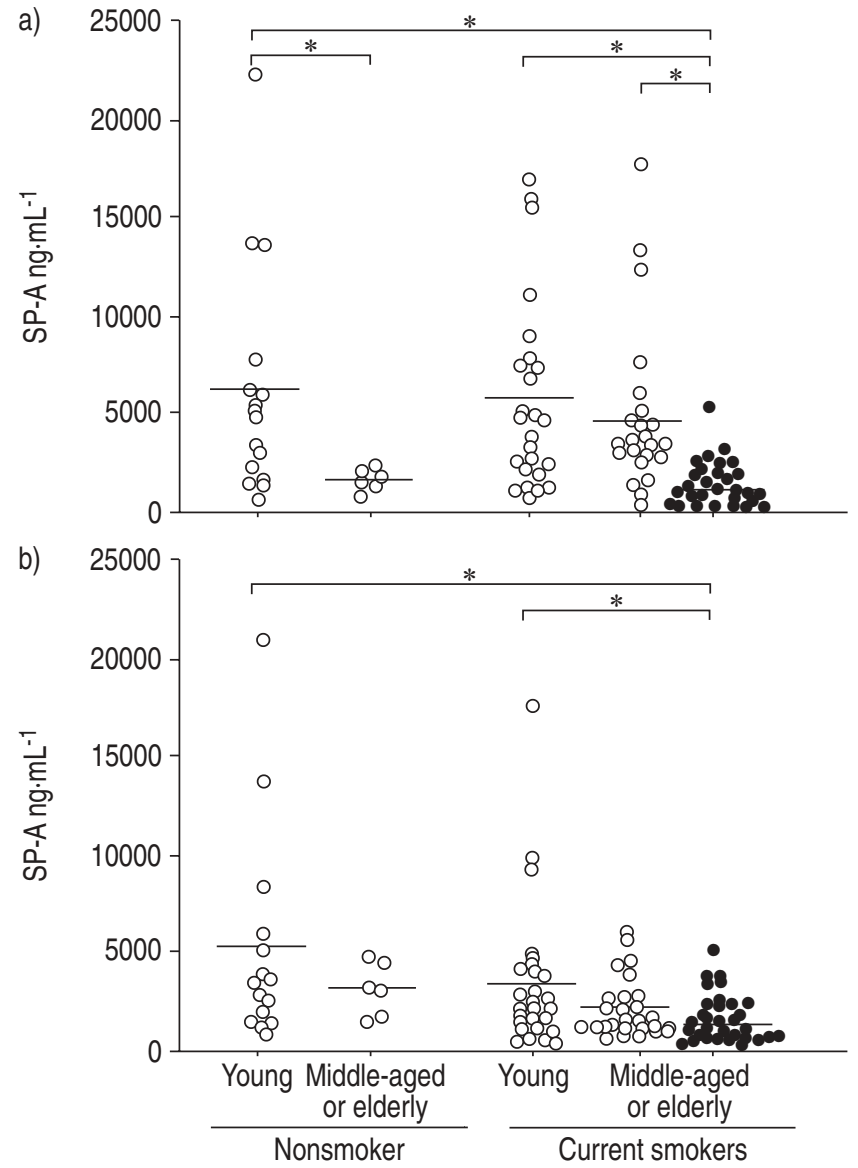

Fig. 1.-Surfactant protein (SP)-A in a) bronchial lavage and b) bronchoalveolar lavage fluid $(\bigcirc$ : without emphysema, $\mathbf{0}$ : with emphysema). Horizontal bars represent mean values. *: $\mathrm{p}<0.05$.

these two forms when compared with BAL fluid from young nonsmokers.

\section{Discussion}

To the best of the authors' knowledge, this is the first study to demonstrate the effects of ageing and long-term smoking on pulmonary collectins in BL and BAL fluids, and to confirm an association between these molecules and emphysema.

Although many studies have been published on the levels of SP-A in lavage fluids from healthy subjects and patients with lung diseases, mean concentrations of SP-A in lavage fluids show great variation [14]. Inconsistent results also have been reported with regard to the effects of smoking on levels of SPA in lavage fluids, which were decreased [15], slightly increased [16], or unchanged [17]. However, no changes were observed in rats exposed to cigarette smoke [18]. Besides differences in the immunochemical measurement of SP-A using different antibodies and standards [19], the large variation and inconsistency of results from different investigators may partially be due to the pre-analytical handling of lavage samples. Because SP-A is tightly bound to phospholipids in a calcium-dependent manner, its concentration in lavage fluids differs markedly when measured in the surfactant phospholipid-rich pellet or in the supernatant of the fluids. KUROKI et al. [12] showed that $99.1 \%$ of SP-A is associated with the crude supernatant pellet and the remaining $0.9 \%$ is present in the supernatant. In this study,

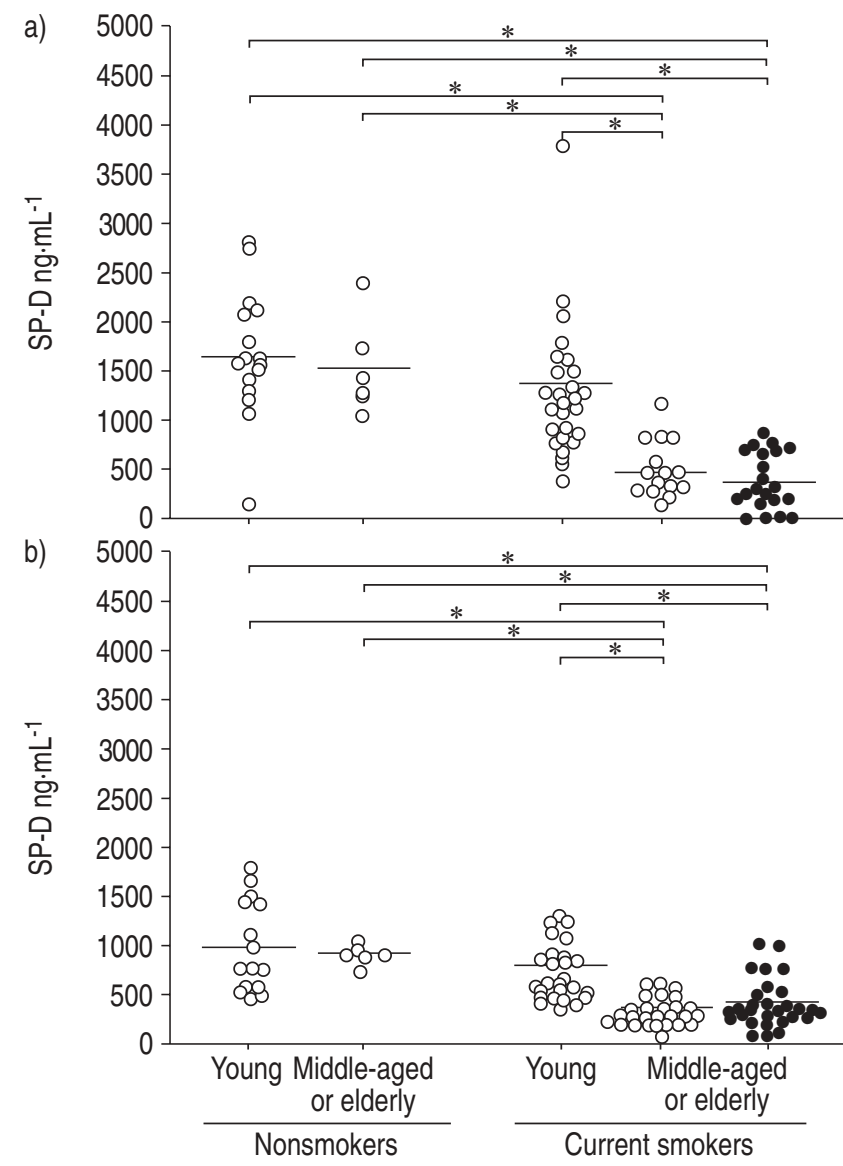

Fig. 2.-Surfactant protein (SP)-D in a) bronchial lavage fluid and b) bronchoalveolar lavage fluid ( $\bigcirc$ : without emphysema, $\bigcirc$ : with emphysema). Horizontal bars represent mean values. *: $\mathrm{p}<0.05$.

vigorous vortexing of each sample was performed in order to avoid technical errors resulting from aggregation and precipitation of SP-A in lavage fluid ex vivo. Pulmonary macrophages are known to bind, incorporate, and degrade SP-A [20], and this capability depends on the state of activation [21]. Increases in the number and/or activation state of macrophages in the lung could be a factor in contributing to the reduced levels of SP-A in epithelial lining fluid in vivo in addition to altered synthesis and secretion of SP-A by pulmonary epithelial cells.

SP-D levels were reportedly low in BAL fluid of smokers [15], although the age factor was not well documented, and thereafter it was speculated that the relative SP-D deficiency in smokers may result in increased numbers of apoptotic cells lingering in the airway and thus contribute to the development of emphysema [22]. SP-D replacement therapy promoted reduction of excess apoptotic cells and reduced inflammation in mice developing emphysema [23]. Although the authors anticipated the relative deficiency of SP-D in the subgroup of smokers developing emphysema based on the findings that SP-D deficiency develops progressive airspace enlargement with age in mice [4], no significant reduction in the levels of SP-D in BL and BAL fluid was observed in association with the presence of emphysema on the HRCT scan. The findings from the current study highlight the difficulty in extrapolating data obtained in animal models to human diseases, at least in part, because the architecture of terminal conducting airways shows species-specific variations in addition to highly diverse epithelial composition [24]. However, it should be noted that emphysema severity was 

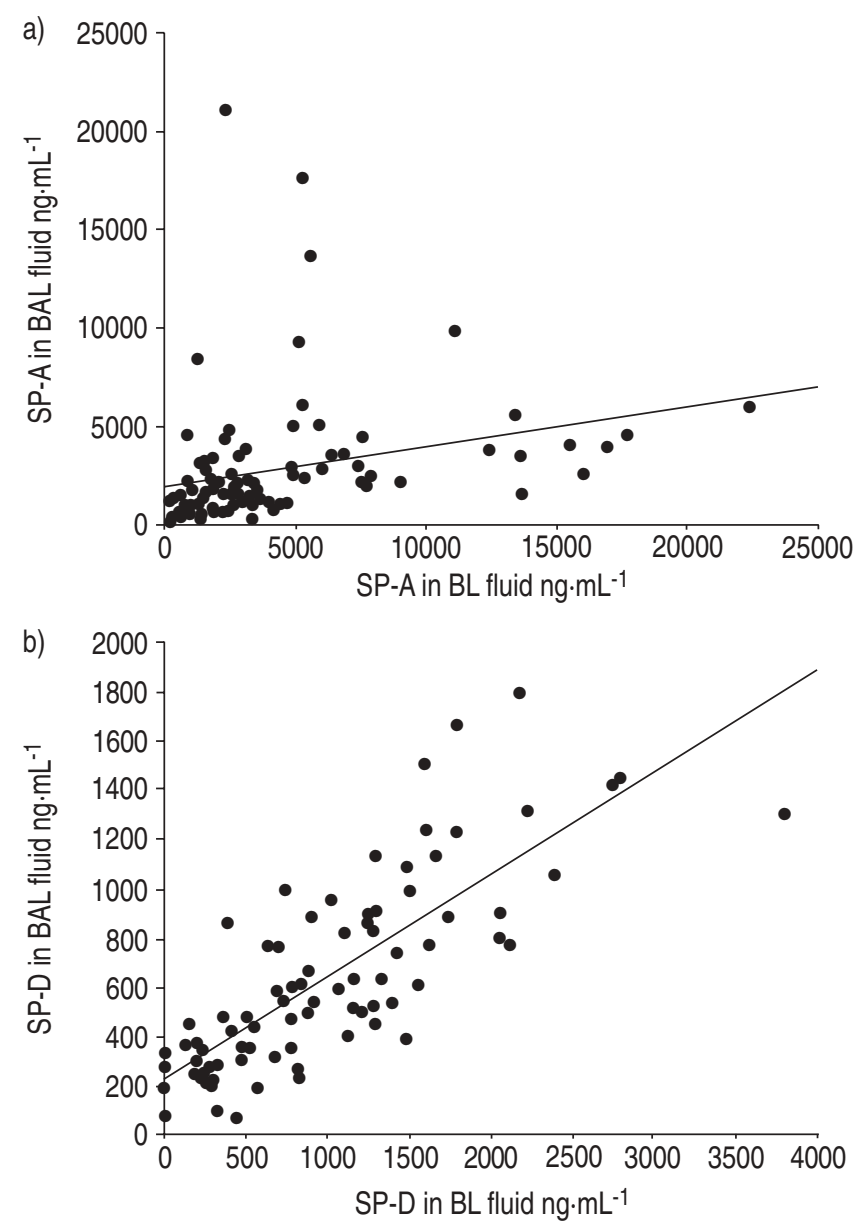

Fig. 3. - Correlation between surfactant protein (SP)-A (a; $n=95$, $\mathrm{p}<0.01, \mathrm{r}=0.07)$ and SP-D $(\mathrm{b} ; \mathrm{n}=82, \mathrm{p}<0.01, \mathrm{r}=0.76)$ levels in bronchial lavage $(\mathrm{BL})$ fluid and bronchoalveolar lavage $(\mathrm{BAL})$ fluid.

classed as low and the distribution was heterogeneous in the subjects in the current study. It thus cannot be ruled out that SP-A and SP-D levels might be lower in individuals with more advanced emphysema. Irrespectively, it might be speculated that decreased SP-A and/or SP-D in the lower respiratory tract, may lead to impaired host defence in the lung, particularly if it is considered that SP-A and SP-D play an important role in innate immunity [2].

Although no pathological correlation was performed in the current study, it is probable that the decrease in SP-A and SP$\mathrm{D}$ in BL and BAL fluids is related to epithelial transformations in the respiratory tract. An increase in goblet cells and squamous metaplasia is a well-established pattern in respiratory bronchioles of smokers, leading to a sparse density of bronchiolar ciliated and nonciliated cells [25], in association to wall inflammation and fibrosis [26]. Therefore, decreases in surfactant proteins in BL and BAL fluids may reflect phenotypic changes in the resident cells or changes in the types of cells located in these anatomical regions. The proteolytic degradation of SP-A and/or SP-D might be an alternative explanation for the decreased levels in lavage fluids because the sandwich ELISA systems used in this study only detects intact SP-A or SP-D, which may form oligomers. CHIBA et al. [27] demonstrated that the epitope for the monoclonal antibodies PC6 and PE10 lies within the region of Thr184-Gly194 located in collagen-like domain (CRD). For SP-D ELISA, the two monoclonal antibodies used

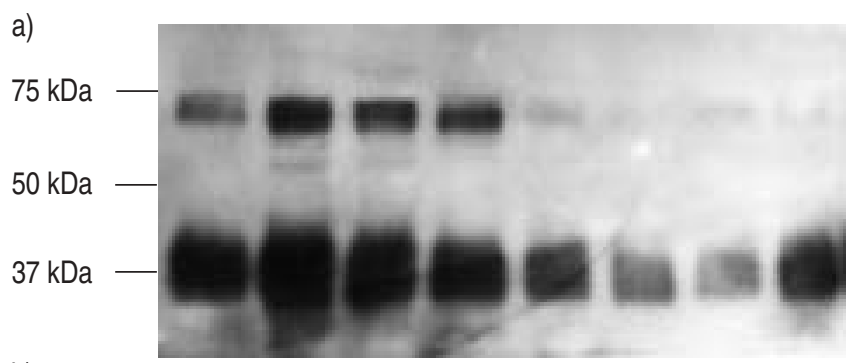

b)

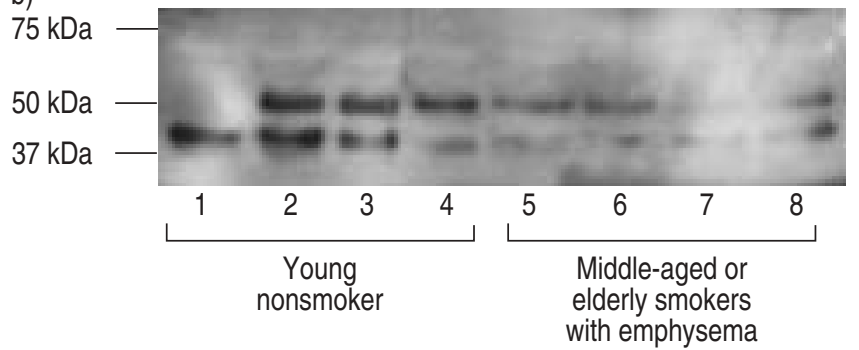

Fig. 4. - Western blotting of surfactant protein (SP)-A and SP-D in bronchoalveolar lavage (BAL) fluid. Representative BAL fluid samples from four young nonsmokers (lanes 1-4) and four middle-aged or elderly current smokers with emphysema (lanes 5-8). Equivalent volume of unconcentrated BAL fluid was loaded in each lane. Molecular weight is indicated on the right. (a) SP-A; two major bands were identified at molecular masses of $\sim 36$ and $62 \mathrm{kD}$. (b) SP$\mathrm{D}$; two major bands were identified at molecular masses of $\sim 43$ and $50 \mathrm{kD}$.

also recognise the common region in CRD (Y. Kuroki, unpublished data). Therefore, using these monoclonal antibodies in ELISA for SP-A or SP-D, only oligomers can be detected because of the possible competition between the two antibodies. MASON et al. [28] demonstrated that type III collagenase directly digests SP-D and produces small fragments. However, dodecamers of SP-D can selectively augment metalloroteinase activity in vitro [29]. The current authors previously reported that interstitial collagenase was significantly elevated in BAL fluid from middle-aged or elderly current smokers with early emphysema [10], in which proteolytic degradation of SP-A and/or SP-D could occur. In order to evaluate the molecular masses of SP-A and SP-D, therefore, Western blotting was performed. No small fragments of SP-A and/or SP-D, which might have undergone various cleavages, were detected in BAL fluid, although the decrease in both SP-A and SP-D at all sizes was confirmed in middle-aged or elderly smokers with emphysema. The presence of $62 \mathrm{kDa}$ band of SP-A in BAL fluid is reported in the reducing condition [5]. The relative loss of this $62 \mathrm{kDa}$ band in middle-aged or elderly smokers is interesting; however, the mechanism is not clear. Further studies are required to elucidate the difference in forms of SP-A in lavage fluids using gel filtration or electron microscopy with shadow technique. A $50 \mathrm{kDa}$-form of SP-D is reportedly a glycosylated variant present in BAL fluid; however, the clinical relevance of this form remains to be elucidated [28]. There is no obvious clinical difference in the subjects between with and without the $50 \mathrm{kDa}$-form of SP-D on the Western blot.

Sampling of the epithelial lining fluid by bronchoalveolar lavage is a common means of studying proteins secreted by the lung epithelium and investigating changes in these proteins in lung diseases [14]. The current authors have performed sequential bronchoalveolar lavage in asymptomatic volunteers with various smoking histories for a series of studies in the First Dept of Medicine, Hokkaido University 
School of Medicine [9, 10]. It has been previously shown that when successive aliquots of lavage fluid are sampled, with initial aliquots representing the proximal airways and later aliquots representing alveolar and distal airway fluid, cell populations vary according to the site of origin in the respiratory tract [30]. Separation of bronchial and distal samples, as performed in this study, tends to highlight these changes, which might occur diversely throughout the airway. In this particular study, there were higher levels of SP-A and SP-D in BL fluid than in BAL fluid. When all the subjects were analysed together, the concentrations of SP-D in BL fluid were closely correlated with those in BAL fluid, while a poor relationship was obtained for SP-A in those fluids, thus suggesting that SP-A in epithelial lining fluid might be regulated in a different manner along the respiratory tract or in part relate to differential losses in association with inflammatory cells. Because of the lack of the appropriate marker of dilution in lavage fluids to correct for variable recovery of epithelial lining fluid [31], the current authors decided not to normalise the data of SP-A and SP-D to albumin or urea, and the data were expressed as the concentrations in the lavaged fluid, as in the other studies $[9,15]$. In the current study, the recovery of lavage fluid was much lower in the middle-aged or elderly smokers, although there was no difference in the level of albumin in BAL fluid among the groups. This may affect the comparisons made between young and middle-aged or elderly smokers and between smokers and nonsmokers among the middle-aged or elderly group.

In summary, the current study demonstrated that longterm smoking markedly decreases surfactant protein-D concentration in the epithelial lining fluid from the lower respiratory tract, regardless of the presence of emphysema, while ageing alone or the combined effects of long-term smoking and emphysema may lead to an obvious decrease of surfactant protein-A. It should be noted that the clinical consequences of decreased levels of surfactant protein-A and surfactant protein-D in epithelial lining fluids along the airway and in alveolar space remain uncertain in vivo. The results from the current study support the concept that acquired surfactant protein-D deficiency due to cigarette smoking may contribute to emphysema in humans, suggesting that surfactant protein-D supplementation may be beneficial. Multifaceted characterisation of middle-aged or elderly smokers with long-term smoking histories, contrasted with young recent smokers, may in part explain the impaired host defence and predisposition of the lungs to destructive lung diseases, such as pulmonary emphysema, which may occur later in life.

\footnotetext{
Acknowledgements. The authors would like to thank R.M. Senior and E.C. Crouch of the Washington University School of Medicine for their helpful comments, and Y. Suzuki for technical assistance in performing Western blotting, and the technical support staff of SRL Inc. for measurement of SP-A.
}

\section{References}

1. Crouch E, Hartshorn K, Ofek I. Collectins and pulmonary innate immunity. Immunol Rev 2000; 173: 52-65.

2. McCormack FX, Whitsett JA. The pulmonary collectins, SPA and SP-D, orchestrate innate immunity in the lung. J Clin Invest 2002; 109: 707-712.

3. Lawson PR, Reid KB. The roles of surfactant proteins A and $\mathrm{D}$ in innate immunity. Immunol Rev 2000; 173: 66-78.
4. Wert SE, Yoshida M, LeVine AM, et al. Increased metalloproteinase activity, oxidant production, and emphysema in surfactant protein D gene-inactivated mice. Proc Natl Acad Sci USA 2000; 97: 5972-5977.

5. Madsen J, Tornoe I, Nielsen O, Koch C, Steinhilber W, Holmskov U. Expression and localization of lung surfactant protein A in human tissues. Am J Respir Cell Mol Biol 2003; 29: 591-597.

6. Madsen J, Kliem A, Tornoe I, Skjodt K, Koch C, Holmskov U. Localization of lung surfactant protein D on mucosal surfaces in human tissues. J Immunol 2000; 164: 5866-5870.

7. Yoshioka A, Betsuyaku T, Nishimura M, Miyamoto K, Kondo T, Kawakami Y. Excessive neutrophil elastase in bronchoalveolar lavage fluid in subclinical emphysema. Am J Respir Crit Care Med 1995; 152: 2127-2132.

8. American Thoracic Society: Medical Section of the American Lung Association. ATS statement - Snowbird Workshop on Standardization of Spirometry. Am Rev Respir Dis 1979; 119: 831-838.

9. Betsuyaku T, Takeyabu K, Tanino M, Nishimura M. Role of secretory leukocyte protease inhibitor in the development of subclinical emphysema. Eur Respir J 2002; 19: 1051-1057.

10. Betsuyaku T, Nishimura M, Takeyabu K, et al. Neutrophil granule proteins in bronchoalveolar lavage fluid from subjects with subclinical emphysema. Am J Respir Crit Care Med 1999; 159: 1985-1991.

11. Shimizu H, Hosoda K, Mizumoto M, et al. Improved immunoassay for the determination of surfactant protein A (SP-A) in human amniotic fluid. Tohoku J Exp Med 1989; 157: 269-278.

12. Kuroki Y, Shiratori M, Ogasawara Y, Tsuzuki A, Akino T. Characterization of pulmonary surfactant protein D: its copurification with lipids. Biochim Biophys Acta 1991; 1086: 185-190.

13. Inoue $\mathrm{T}$, Matsuura E, Nagata A, et al. Enzyme-linked immunosorbent assay for human pulmonary surfactant protein D. J Immunol Methods 1994; 173: 157-164.

14. Hermans C, Bernard A. Lung epithelium-specific proteins: characteristics and potential applications as markers. $\mathrm{Am} \mathrm{J}$ Respir Crit Care Med 1999; 159: 646-678.

15. Honda $\mathrm{Y}$, Takahashi $\mathrm{H}$, Kuroki $\mathrm{Y}$, Akino $\mathrm{T}$, Abe $\mathrm{S}$. Decreased contents of surfactant proteins A and D in BAL fluids of healthy smokers. Chest 1996; 109: 1006-1009.

16. Hamm H, Luhrs J, Guzman y Rotaeche J, Costabel U, Fabel $\mathrm{H}$, Bartsch W. Elevated surfactant protein A in bronchoalveolar lavage fluids from sarcoidosis and hypersensitivity pneumonitis patients. Chest 1994; 106: 1766-1770.

17. Lesur $\mathrm{O}$, Bernard $\mathrm{A}$, Arsalane $\mathrm{K}$, et al. Clara cell protein (CC-16) induces a phospholipase A2-mediated inhibition of fibroblast migration in vitro. Am J Respir Crit Care Med 1995; 152: 290-297.

18. Subramaniam S, Whitsett JA, Hull W, Gairola CG. Alteration of pulmonary surfactant proteins in rats chronically exposed to cigarette smoke. Toxicol Appl Pharmacol 1996; 140: 274-280.

19. Phelps DS, Umstead TM, Mejia M, Carrillo G, Pardo A, Selman M. Increased surfactant protein-A levels in patients with newly diagnosed idiopathic pulmonary fibrosis. Chest 2004; 125: 617-625.

20. Bates SR, Fisher AB. Surfactant protein A is degraded by alveolar macrophages. Am J Physiol 1996; 271: L258L266.

21. Bates SR, Xu J, Dodia C, Fisher AB. Macrophages primed by overnight culture demonstrate a marked stimulation of surfactant protein A degradation. Am J Physiol 1997; 273: L831-L839.

22. Clark H, Reid K. The potential of recombinant surfactant protein $\mathrm{D}$ therapy to reduce inflammation in neonatal chronic lung disease, cystic fibrosis, and emphysema. Arch Dis Child 2003; 88: 981-984. 
23. Clark H, Palaniyar N, Strong P, Edmondson J, Hawgood S, Reid KB. Surfactant protein D reduces alveolar macrophage apoptosis in vivo. J Immunol 2002; 169: 28922899.

24. Plopper C. Clara cells. In: McDonald JA, eds. Lung growth and development. New York, Dekker, 1997; pp. 181-209.

25. Lumsden AB, McLean A, Lamb D. Goblet and Clara cells of human distal airways: evidence for smoking induced changes in their numbers. Thorax 1984; 39: 844-849.

26. Saetta M, Turato G, Baraldo S, et al. Goblet cell hyperplasia and epithelial inflammation in peripheral airways of smokers with both symptoms of chronic bronchitis and chronic airflow limitation. Am J Respir Crit Care Med 2000; 161: 1016-1021.

27. Chiba H, Sano H, Saitoh M, et al. Introduction of mannose binding protein-type phosphatidylinositol recognition into pulmonary surfactant protein A. Biochemistry 1999; 38: 7321-7331.

28. Mason RJ, Nielsen LD, Kuroki Y, Matsuura E, Freed JH, Shannon JM. A 50-kDa variant form of human surfactant protein D. Eur Respir J 1998; 12: 1147-1155.

29. Trask BC, Malone MJ, Lum EH, Welgus HG, Crouch EC, Shapiro SD. Induction of macrophage matrix metalloproteinase biosynthesis by surfactant protein D. J Biol Chem 2001; 276: 37846-37852.

30. Martin TR, Raghu G, Maunder RJ, Springmeyer SC. The effects of chronic bronchitis and chronic air-flow obstruction on lung cell populations recovered by bronchoalveolar lavage. Am Rev Respir Dis 1985; 132: 254-260.

31. Marcy TW, Merrill WW, Rankin JA, Reynolds HY. Limitations of using urea to quantify epithelial lining fluid recovered by bronchoalveolar lavage. Am Rev Respir Dis 1987; 135: 1276-1280. 